\title{
To err is human
}

\begin{abstract}
While we all make mistakes almost every day of our lives, we try to keep those errors to a minimum—at least in the pages of our journals. Here are a few ways in which Nature journals try to keep the creep of errors at bay.
\end{abstract}

$\mathbf{T}$ he breadth and depth of the internet has made plagiarism all too tempting. You read a few perfectly phrased sentences, and in a moment they are cut and pasted into a Word document. You forget to note where the phrases came from, thinking that you'll remember to do it later, and you continue to work on your paper. Days (or months) later, you have no recollection of ever having lifted that paragraph from another paper, and you are ready to submit your manuscript to a journal.

Before we describe what we do from our end, what is the definition of plagiarism? According to the Office of Research Integrity (ORI), which handles complaints in biomedicine, plagiarism is the "appropriation of another person's ideas, processes, results, or words without giving appropriate credit."

Over the past year, the Nature Research Journals, including NSMB, have each been checking about two papers a month for identical or paraphrased passages against previously published articles. We have used CrossCheck (http://www.crossref.org/crosscheck.html), a plagiarism-checking service that uses iThenticate (http://crossref. ithenticate.com/), a plagiarism software program. We have also used CrossCheck in cases of suspected plagiarism. The good news is that, across all the journals, no cases were detected during the year-long trial. We will continue to spot-check random articles in this fashion, unless we have reason to be suspicious.

When it comes to figures and images, we all want those many hours of work to be represented in as good an image as possible. But beware: a 'picture-perfect' image could land you in trouble, no matter how innocent your intensions.

After some consultation with experts, the Nature family of journals has come up with a guide for how to handle digital images (http://www. nature.com/nature/authors/submissions/images/). In brief, the less you do, the better. Some image processing is acceptable, but it must be applied equally to the entire image, and the image-acquisition tools and image-processing software packages must be clearly indicated. The use of any touch-up tools to manipulate the image is not allowed. Cropped images of gels or blots must be clearly separated so that it is clear that the lanes were not contiguous in the experiment, and the cropping must be clearly explained in the figure legend. In addition, whenever possible, the Supplementary Information should include the full-length gels and blots. All our images and figures are examined by our production editors, but only those that raise red flags (and I am being purposefully vague here) are scrutinized more closely for image manipulation. When such cases are identified, we contact the authors for clarification and explanation to resolve any potential problems before publication. Thus far, we have been able to resolve any issues that have come up.

Most recently, the Protein Data Bank (PDB) has asked all journals that publish structures to consider partnering with it to adopt a new procedure that will give editors and referees access to PDB validation reports that are generated automatically as part of the PDB deposition and annotation process. These reports summarize the results of various geometric and experimental data checks that are performed using community-established standards and software.

The PDB validation reports provide critical information that should enable editors and referees to better evaluate the quality of a structure described in a submitted manuscript, while keeping the coordinate file confidential. With these reports in hand, editors and referees could raise issues of concern to authors so that those issues can be addressed before the structure is published.

But before considering making these PDB validation reports part of the review process, there are important questions to consider:

- Would you as an author be willing to deposit coordinates at submission so that these reports could be generated as soon as possible and provided both to the reviewers and the editors? The PDB says that they can typically generate the report in about two weeks.

- If not, when would you be willing to deposit coordinates? At present, journal policy insists that coordinates be deposited when the article is accepted in principle and that they are released upon publication.

- How valuable would these reports be to you as an author? And how valuable would they be to you as a reviewer?

- As a reader, would you want to see the reports published as part of the Supplementary Information?

Answers to these questions, as well as additional comments, are welcome, and we look forward to hearing from as many of you as possible at nsmb@us.nature.com. 